\title{
DESIGN AND ANALYSIS OF UNDERTRAY DIFFUSER FOR A FORMULA STYLE RACECAR
}

\author{
Ali Asgar S. Khokhar', Suhas S. Shirolkar² \\ ${ }^{1}$ Graduate in Mechanical Engineering, KJ Somaiya College of Engineering, Mumbai, India. \\ Email: khokharali5252@gmail.com, Contact:+91-8655397386 \\ ${ }^{2}$ Graduate in Mechanical Engineering, JSS Academy of Technical Education, Bangalore, India \\ Email: suhas.shirolkar@gmail.com,Contact: +91-9739554154
}

\begin{abstract}
The advancements in Formula one industry have clearly shown the importance of Aerodynamics and thus it was taken as an opportunity to design and develop a not much widely known aerodynamic component, a diffuser considering the myriad of benefits. This report explains the development of an undertray diffuser for an Formula Student (fsae) car. An undertray Diffuser is just as the front and back wing of race cars an aerodynamic package that generates Downforce. The hard part of designing an aerodynamic package for these cars is their top speed. The faster a car drives the more downforce is going to be generated. The Formula student race cars have a top speed around $130 \mathrm{~km} / \mathrm{h}$. Due to this low top speed (Formula 1 cars reach top speeds of $370 \mathrm{~km} / \mathrm{h}$ ), the wings of the car have to work with lower speeds and have to be larger. The undertray diffuser has to generate as much downforce as possible and as less drag as possible. The working principle of the undertray diffuser is explained later. The air under the undertray diffuser travels faster than on top of the undertray diffuser. When this happens a lower pressure is generated underneath the undertray diffuser and this lower pressure generates downforce.
\end{abstract}

Keywords: Aerodynamics, downforce, speed, pressure.

\section{INTRODUCTION}

Down force is known as the vertical force that is applied on the vehicle which is not because of the mass, but because of the air flowing around it. Due to this force, the tire's coefficient of friction may decrease. However, efficiently designed aerodynamic components giving the tires more grip and hence better acceleration. In formula racing, these components are mainly the wings and undertray diffusers. From literature we understand that, efficient under body diffuser produces maximum down force with minimum or negligible drag. While on the other hand wings (both front and rear) produces comparatively more down force with a disadvantage of noteworthy drag and weight addition

\section{WORKING PRINCIPLE:}

The concept used in designing a diffuser is the ground effect, that is, to cause a venturi-like effect under the vehicle. Under such a vehicle, there is a nozzle that proliferates the velocity of the air below the vehicle and a throat is formed where the maximum velocity exists and then a component called undertray slows this air back down to free stream velocity. As per Bernoulli's Equation, we know that when the local velocity increases, the local pressure is decreased. Because of this lower pressure under the vehicle and the higher pressure on top, a force called downforce is applied on the vehicle.

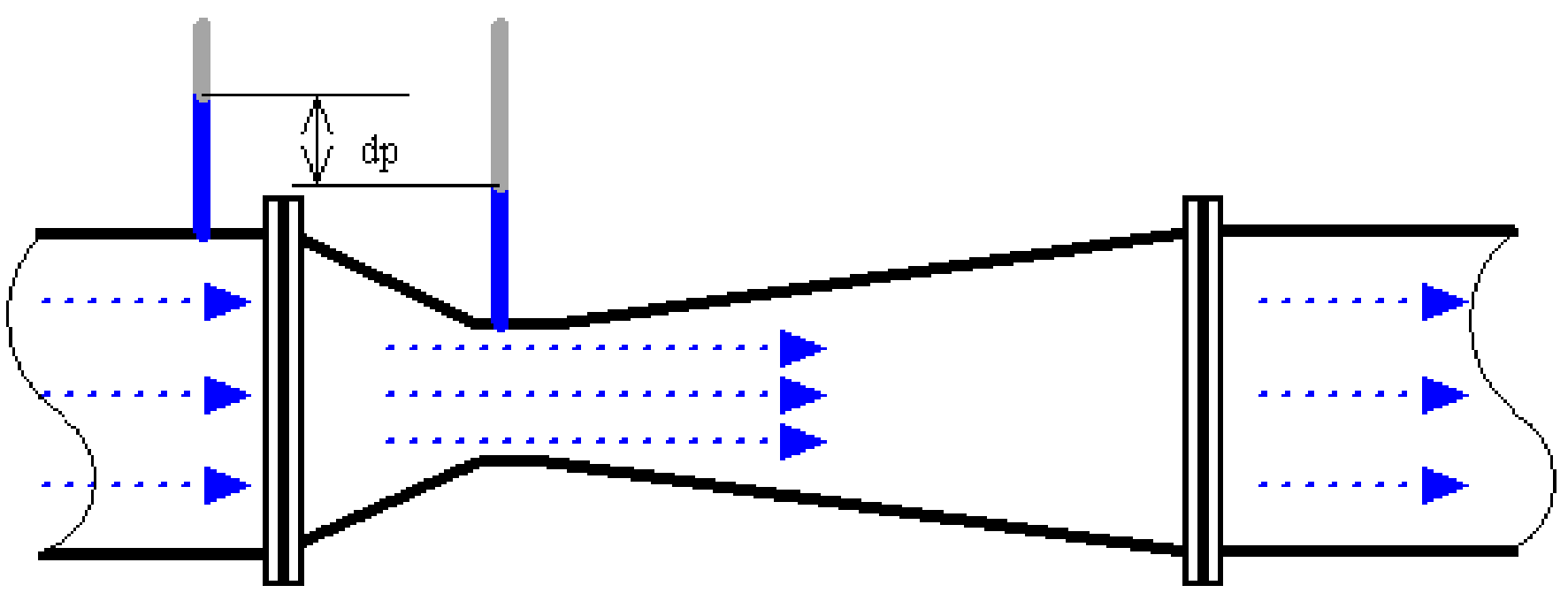

Figure 1: Venturi Tube 


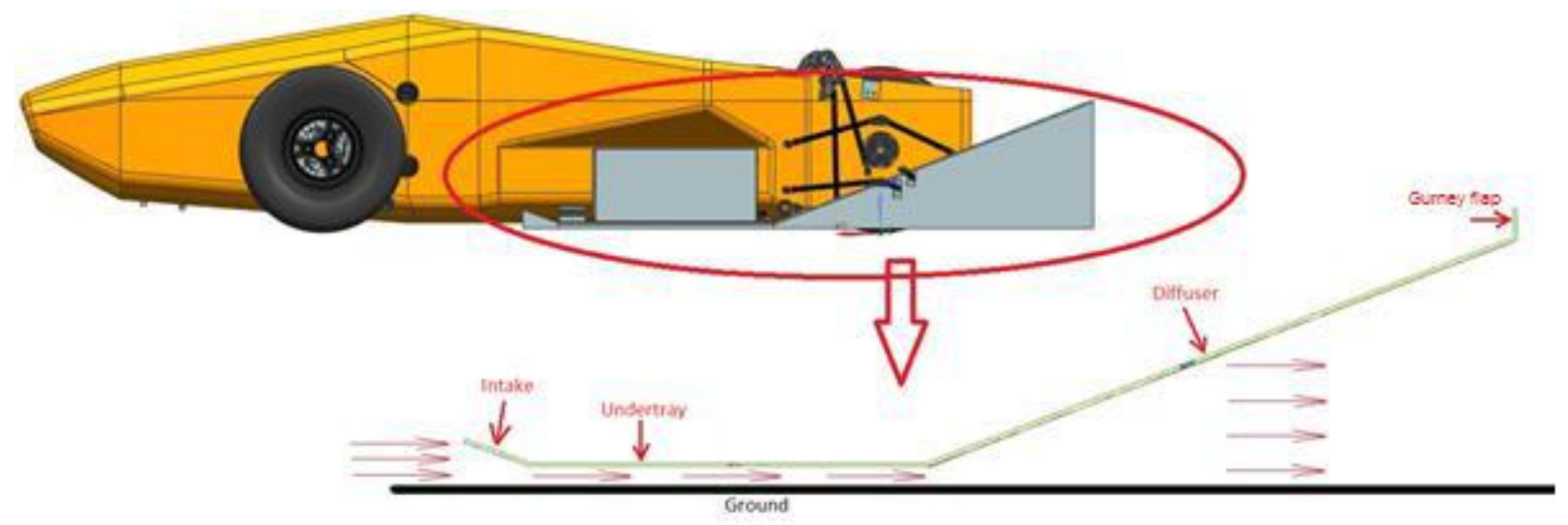

Figure 2: Undertray Diffuser (representative picture)

The efficiency of the whole component purely depends on the efficiency of the diffuser section. The main role of the diffuser is to slow the air flowing under the vehicle and thus reduce it to the free stream which subsides the drag and increase the overall undertray efficiency..

The main moto while designing is to get the highest possible angle without flow separation as incase there is a separation, it may lead to more drag and thus lesser downforce.

There are a few more factors that will make a difference in downforce and/or drag.

\section{- Ground clearance:}

When there is a large ground clearance, the proportional cross-sectional area will not reduce much from the start of the intake until the end of the intake. So when the ground clearance will be made very small the air will move faster than with a larger cross-sectional surface.

With respect to this fact the ground clearance has to be very small, but this will give two problems. The first problem is that when the ground clearance is too small, the undertray will produce positive lift since there is hardly any airflow between the undertray and the ground. The second problem is that due to irregularities at the surface of the ground, the undertray could hit the ground when the ground clearance is too small. So the ground clearance cannot be too large and not too small.

- $\quad$ The angle of the diffuser:

If the angle of the diffuser is close to zero the boundary layer flow will not detach, but the air speed will not be reduced enough to make a laminar transition of the air at the end of the car when to two airstreams meet.

If the diffuser has a very large angle the boundary layer flow will detach and the airflow underneath the diffuser will be turbulent.

So the angle of the diffuser is limited with two boundaries: when the angle is too small or too large angle the diffuser will create more drag then necessary.

\section{- $\quad$ Air speed:}

With an increasing driving speed the airspeed will also increase. When this happens the proportional difference between the speed of the air on top of the undertray and underneath the undertray will get higher. This means the difference in pressure will be higher.

So the generated downforce and drag are completely dependent on the dimensions of the undertray diffuser and the speed of the air/speed of the car. When the incorrect dimensions are taken the undertray diffuser will generate a lot of drag and will generate a little amount of downforce or even positive lift.

Considering these factors, a diffuser can be developed with minimum drag and thus maximum downforce with proper CFD analysis.

\section{COMPUTATIONAL FLUID DYNAMICS :}

CFD basically helps in stimulating the real time dynamic scenario..

With the various inputs from the literature, a basic model was developed so that there could be a platform to start working from and derive optimum values of lift, drag and center of pressure location. CFD analysis was used as a narrowing tool to filter the variables and obtain a better downforce.

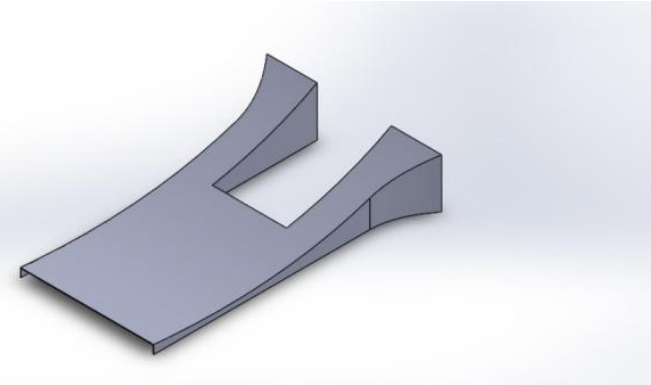

Figure 3: Under tray diffuser Iteration 1

Various parameters which were considered while designing the undertray diffuser which are as follows:

1. Inlet angle.

2. Outlet angle.

3. Starting point. 

4. Other dimensions.
5. Ground clearance.
6. Velocity.

\section{RESULTS OF CFD ANALYSIS: \\ a. OUTLET ANGLE:}

The outlet angle of the diffuser plays an important role in terms of downforce as well as drag. If the angle of the diffuser is close to zero the boundary layer flow will not detach, but the air speed will not be reduced enough to make a laminar transition of the air at the end of the car when to two airstreams meet. If the diffuser has a very large angle the boundary layer flow will detach and the airflow underneath the diffuser will be turbulent. So the angle of the diffuser is limited with two boundaries: when the angle is too small or too large angle the diffuser will create more drag then necessary.

To find out the most preferable outlet angle where we can achieve maximum downforce yet the flow is not separated, we analysed the basic model by changing the angle form 11 degree to 21 degree.

From the analysis we found that with increase in outlet angle there is increase in down force with a decrease in drag. thus the drag/ downforce ratio decreases.
Drag/ Downforce ratio for various outlet angles are:

Table1: Outlet angles

\begin{tabular}{|l|l|}
\hline Outlet Angle & Drag/ Downforce ratio \\
\hline 11 & 0.0121 \\
\hline 14 & 0.0115 \\
\hline 18 & 0.0109 \\
\hline 21 & 0.0106 \\
\hline
\end{tabular}

Thus minimum drag to downforce ratio was found for 21 degree outlet angle. Also comparing the contours of pressure and velocity for all the angles, the velocity was peak at approx $24 \mathrm{~m} / \mathrm{s}$ over entire length of the undertray which is not the case with other angles. Also there was minimum pressure of $101119.48 \mathrm{~Pa}$ achieved at the undertray region. The outlet velocity of air coming out from diffuser is approx $15.45 \mathrm{~m} / \mathrm{s}$ which closely resembles to inlet condition of $18 \mathrm{~m} / \mathrm{s}$

Below are some figures representing pressure and velocity plot due to change in outlet angle.

\section{$>$ Outlet angle -11 degree}

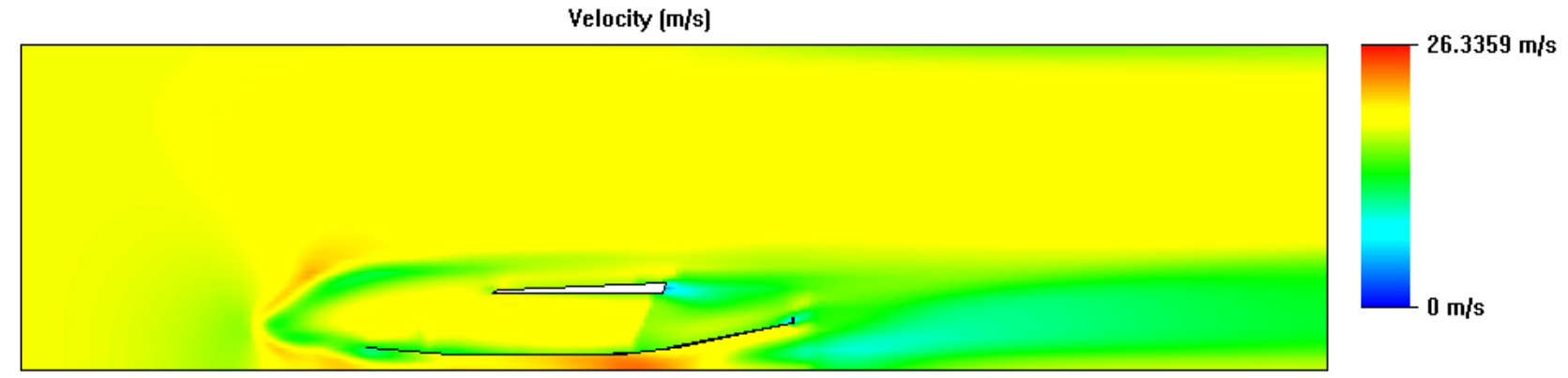

Min $=0 \mathrm{~m} / \mathrm{s} \quad$ Max $=26.3359 \mathrm{~m} / \mathrm{s}$ Iteration $=\mathbf{2 4 7}$

Figure 4: Velocity plot showing side view

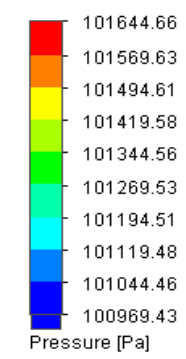

Flow Trajectories 1

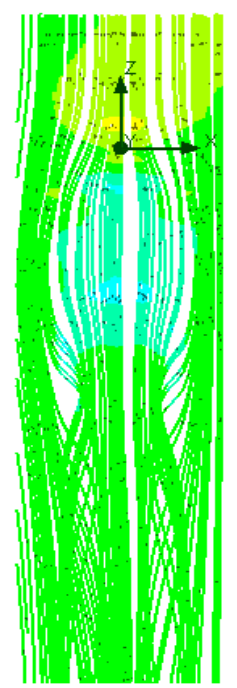


$>$ Outlet Angle - 14 degree
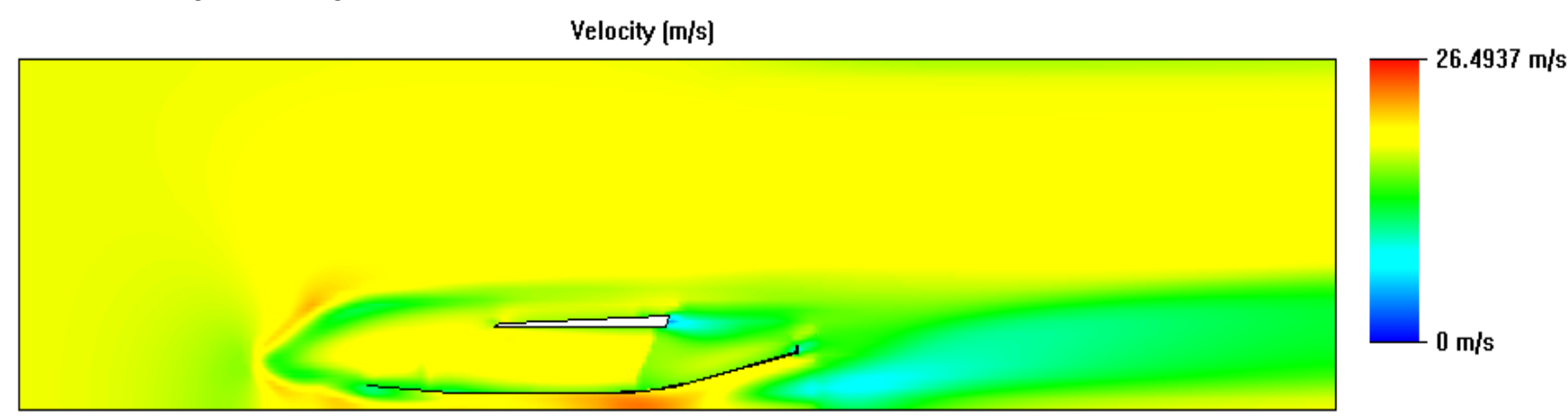

Min $=0 \mathrm{~m} / \mathrm{s} \quad$ Max $=26.4937 \mathrm{~m} / \mathrm{s}$

Iteration $=154$

Figure 5 : Velocity plot
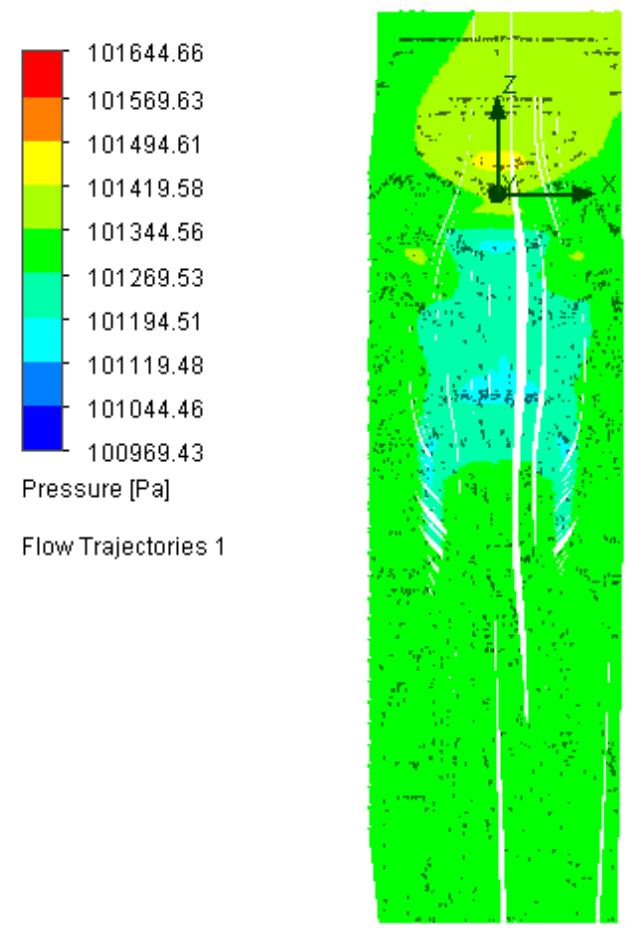

Outlet Angle - 18 degree

Flow Trajectories 1

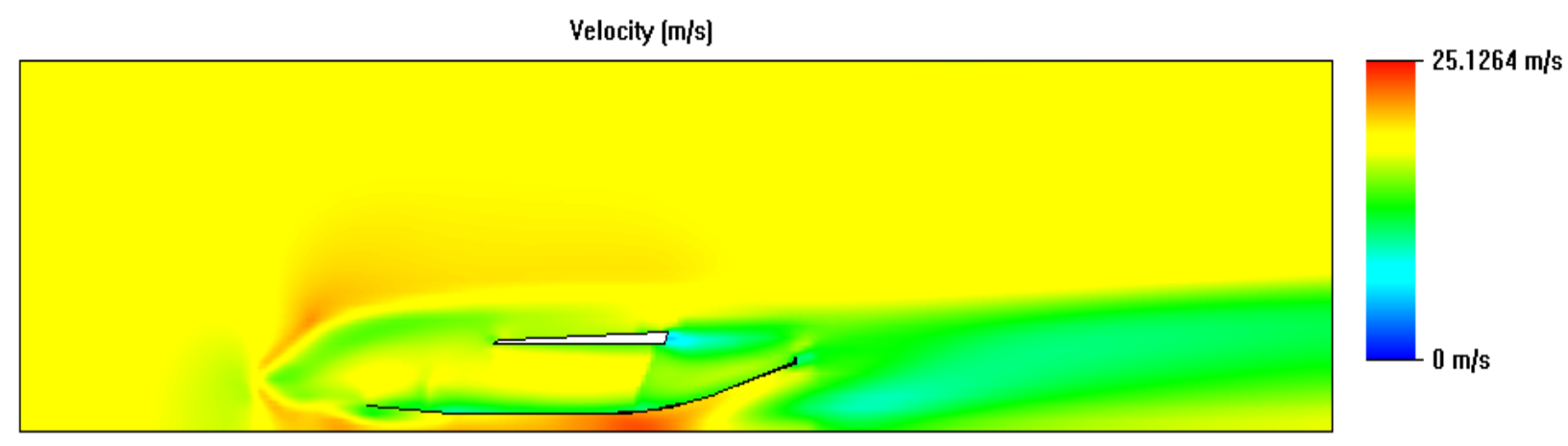

Min=0 m/s Max $=25.1264 \mathrm{~m} / \mathrm{s}$

Iteration $=136$

Figure 6 : Velocity plot 

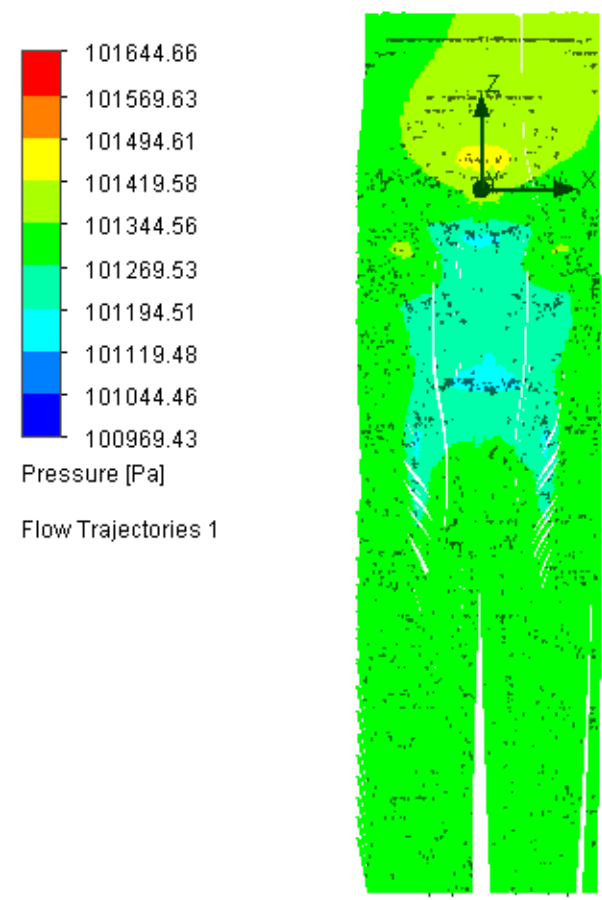

Outlet Angle - 21 degree.

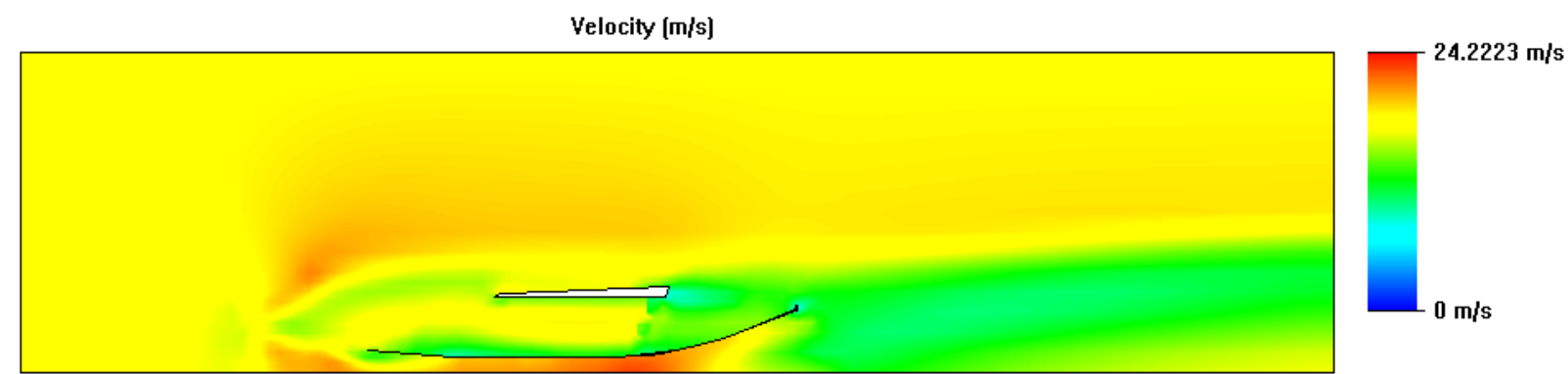

Min $=0 \mathrm{~m} / \mathrm{s} \quad$ Max $=24.2223 \mathrm{~m} / \mathrm{s}$

Iteration $=\mathbf{1 3 3}$

Figure 7: Velocity plot

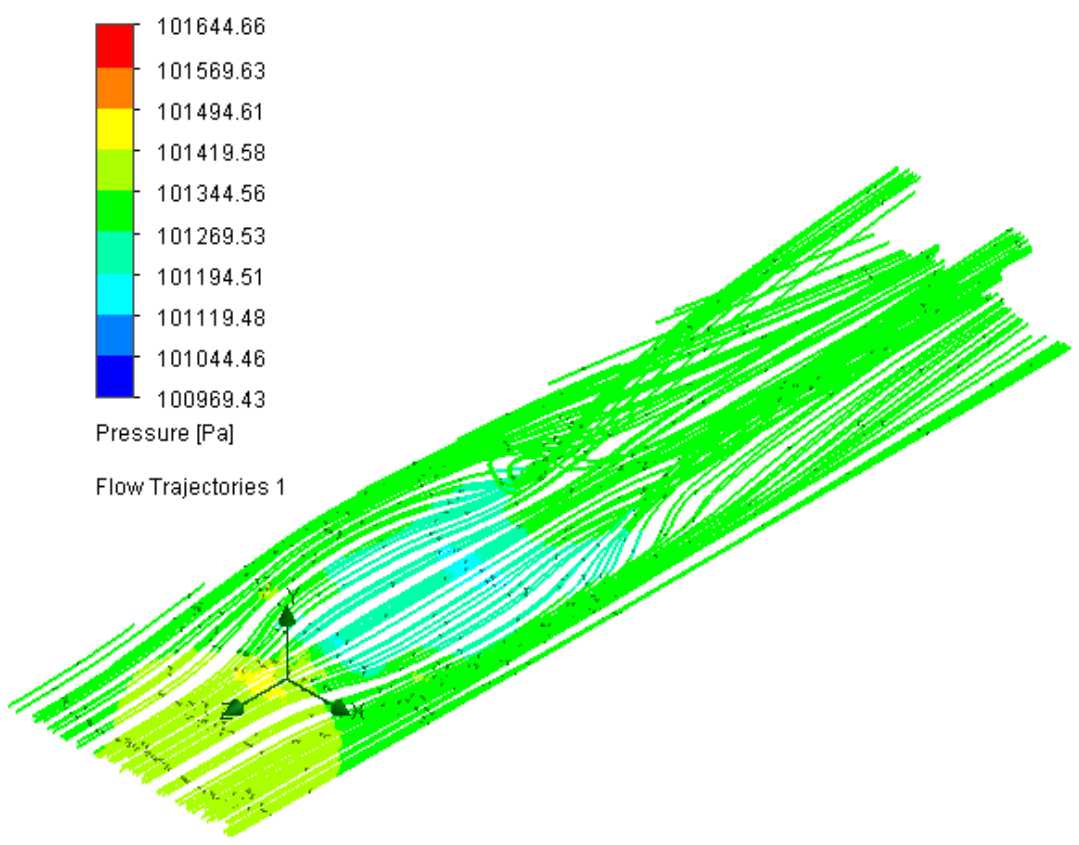




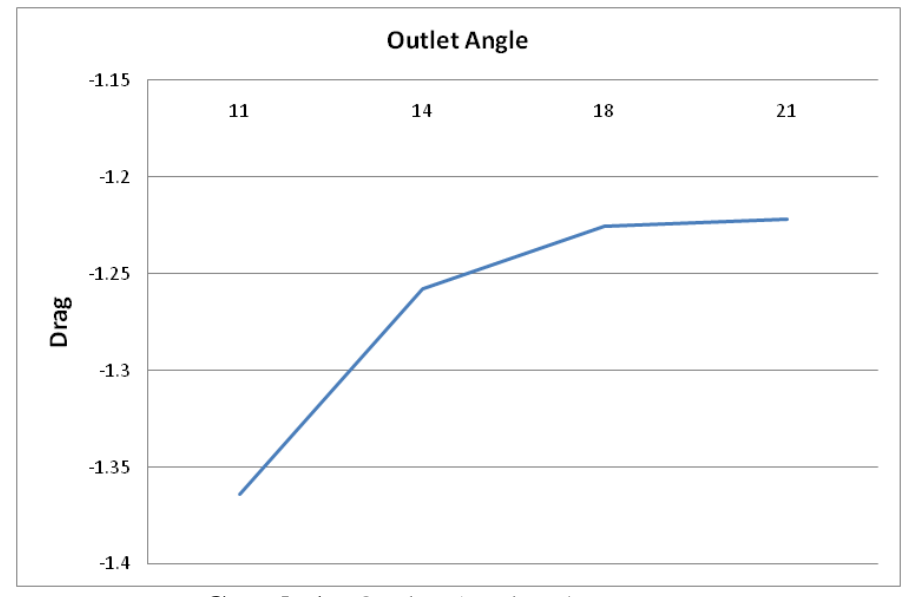

Graph 1: Outlet Angle v/s Drag

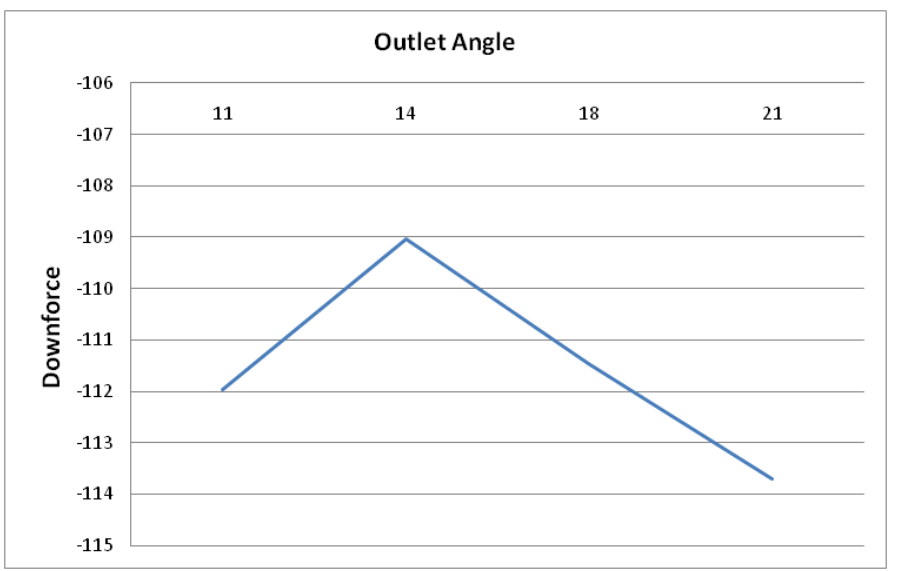

Graph 2: Outlet Angle v/s Downforce

\section{b. INLET ANGLE:}

Inlet angle of the diffuser plays an important role as it acts as a nozzle and channels the air below the undertray. This nozzle increases the velocity of the air while passing through it. If this angle is zero there is no increase in velocity formed. But if this angle is very high then the it would be very difficult to make the outlet conditions similar to inlet condition and will require larger area for it. Also there is restriction in dimension as well.

Inlet angle of the undertray was analysed for 1 degree to 5 degree.

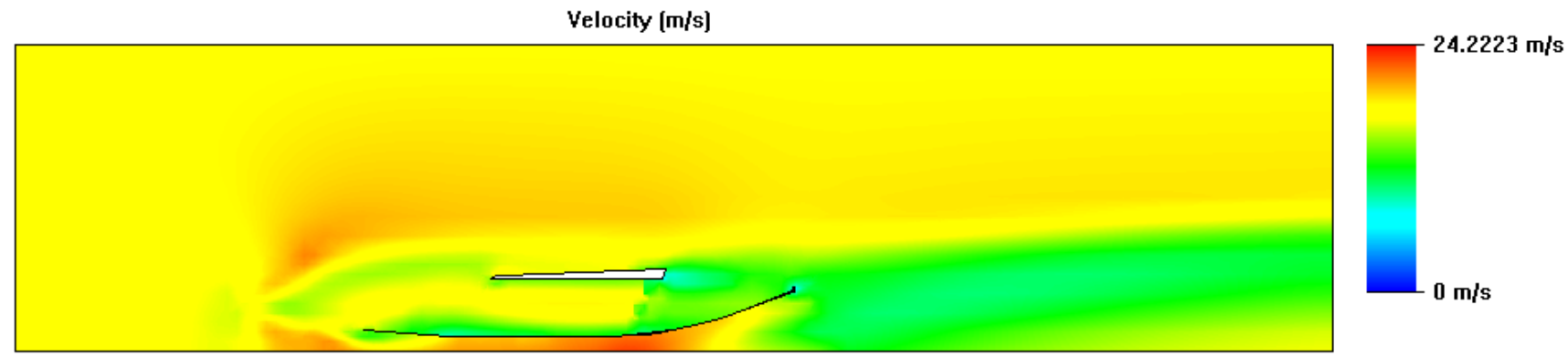

$\operatorname{Min}=0 \mathrm{~m} / \mathrm{s} \quad \operatorname{Max}=24.2223 \mathrm{~m} / \mathrm{s}$

Iteration $=\mathbf{1 3 3}$

Figure 8: Velocity plot at 4 degree inlet and 21 degree outlet

\section{Dimensions:}

To integrate the undertray properly with the diffuser, the dimensions have to be modifies accordingly such as to avoid the jacking bar etc. 


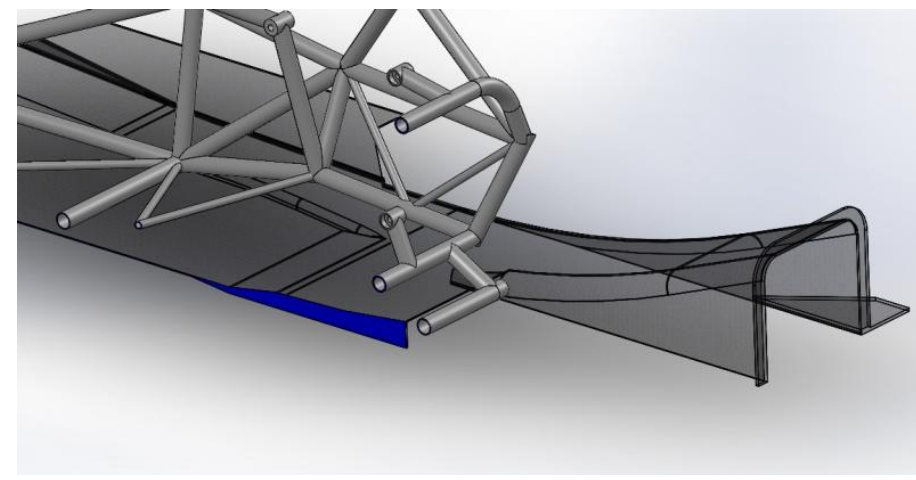

Figure 9: Cut section of Diffuser

\section{c. GROUND CLEARANCE:}

After the dimensions and angles of the diffuser were finalized, the final iteration was then analysed for variation in ground clearance. To study the ground effect with change in ground clearance, downforce and drag was plotted for 30 $\mathrm{mm}$ to $55 \mathrm{~mm}$.

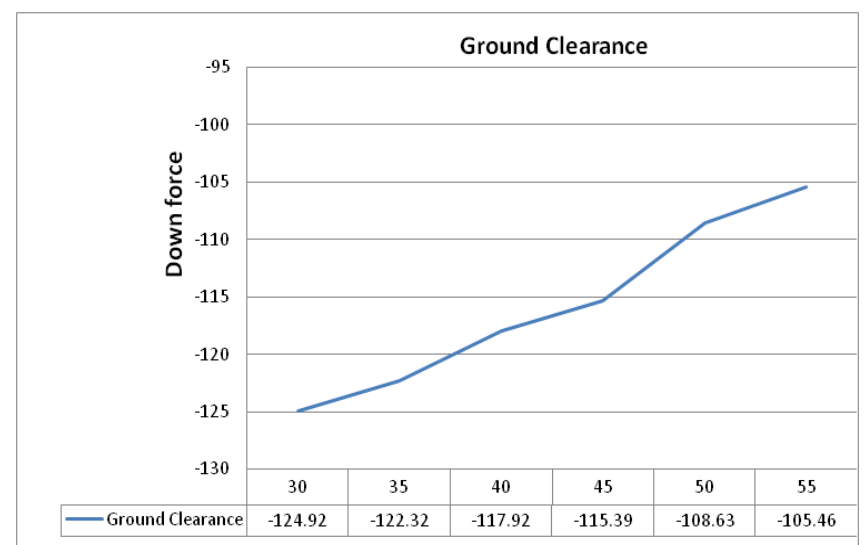

Graph 3: Ground Clearance v/s Downforce

Rules state that there should be minimum ground clearance of 1 inch ( $25.40 \mathrm{~mm}$ ) Thus we decided to keep the ground clearance as $40 \mathrm{~mm}$. Although we can lower the ride height even more, we decided to work with $40 \mathrm{~mm}$ keeping Indian driving condition in consideration. All the further analysis were made assuming the ground clearance to be $40 \mathrm{~mm}$.

\section{d. VELOCITY}

During the competition car will run at various velocity .

Thus to understand the effect of velocity on downforce and diffuser, the undertray was tested out for velocity from 16 $\mathrm{m} / \mathrm{s}$ to $26 \mathrm{~m} / \mathrm{s}$.

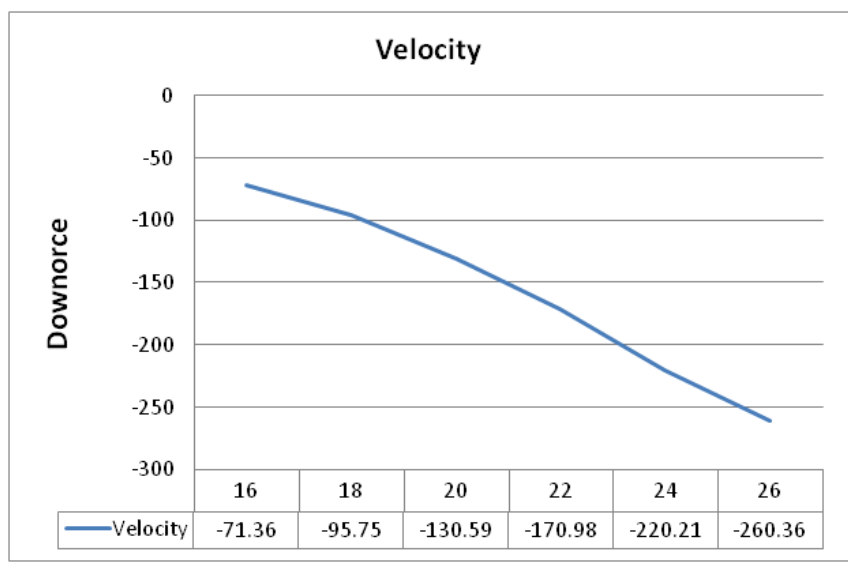

Graph 4: Velocity v/s Downforce

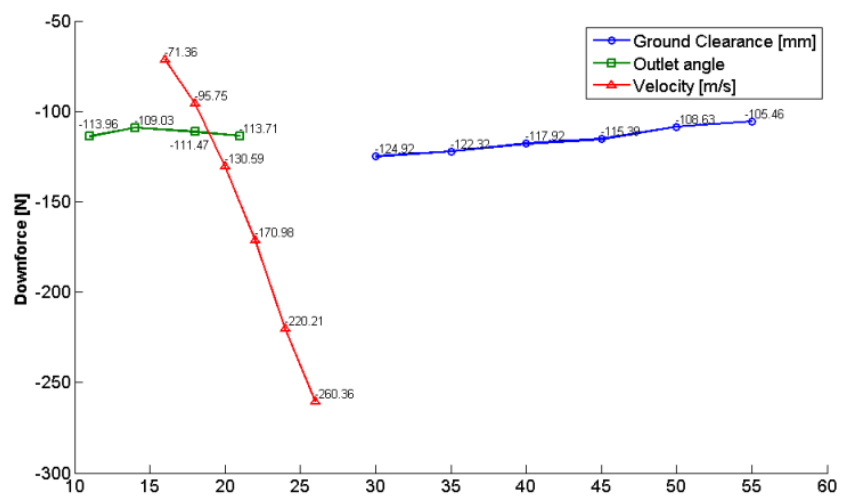

\section{e. Flow Distribution:}

To ensure actual dynamic conditions, analysis was done considering complete vehicle with diffuser mounted.

The model was analysed with the boundary conditions of 18 $\mathrm{m} / \mathrm{s}$ velocity and $40 \mathrm{~mm}$ ground clearance. 


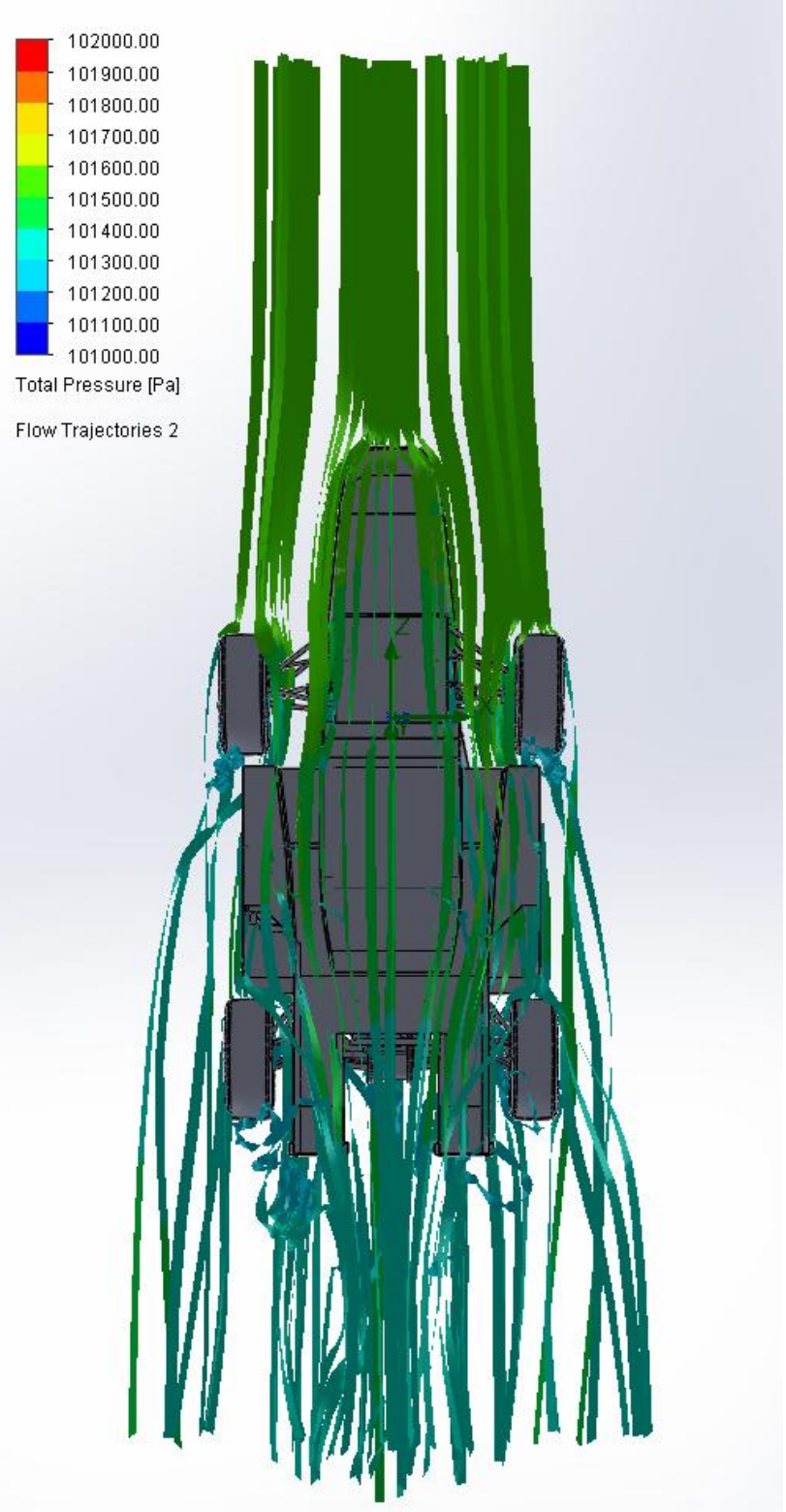

Figure 9: Pressure flow lines in bottom

In the surface pressure plot in the bottom major low pressure region is generated in the undertray diffuser region which is 101114.29 Pa. Rest of the diffuser is subject to a pressure of 101228.57. This justifies the generation on downforce and effectiveness of the diffuser.

Unsymmetrical pressure distribution at left side of the undertray is because of the radiator on the top of it.

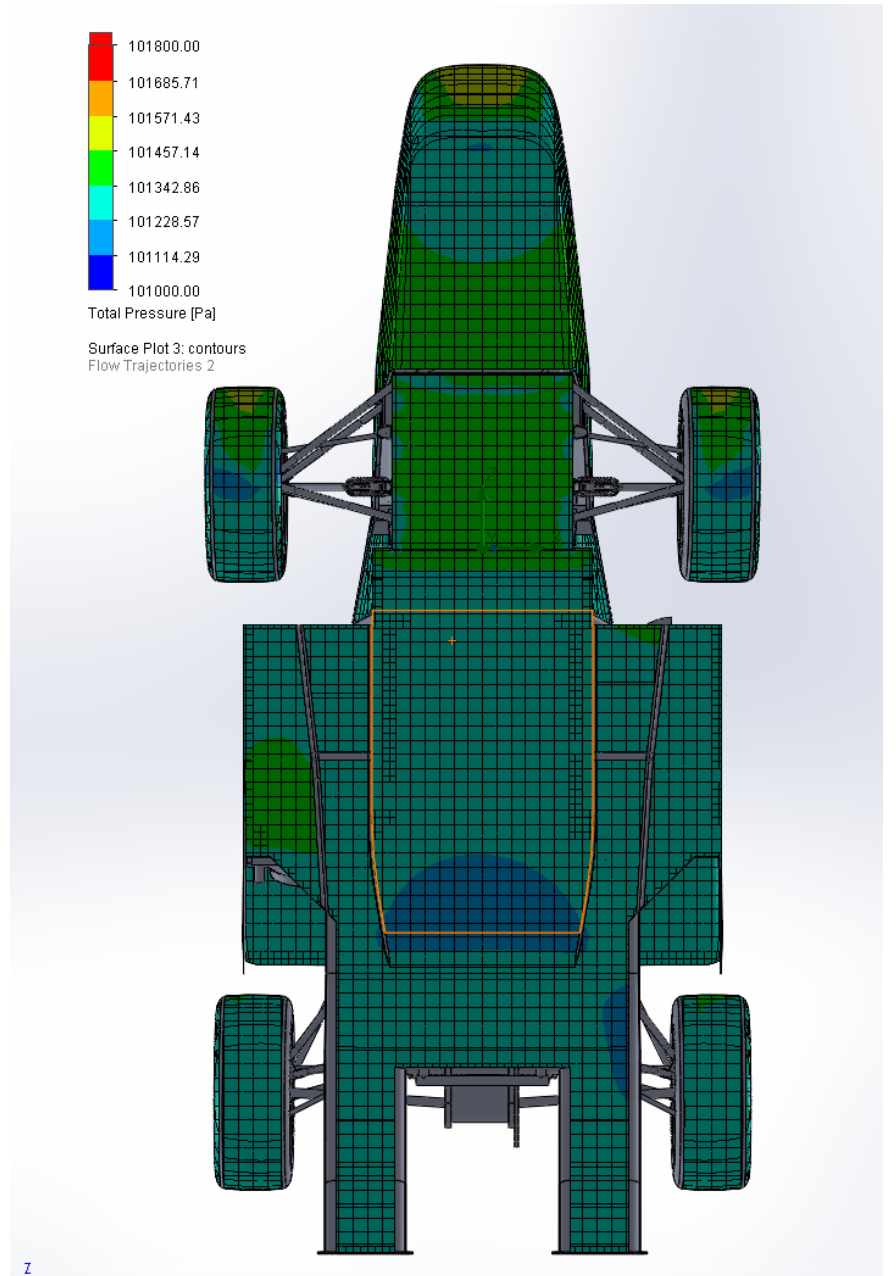

Figure 10: Bottom surface pressure plot

\section{CONCLUSION:}

Thus, we developed the undertray that produces maximum down force with minimum or negligible drag and thus proving its eminence over the wings (front and rear) that even though produce comparatively more down force but with a disadvantage of noteworthy drag and weight addition. This model would be manufactured using the Vacuum infusion technique using Carbon fibre and epoxy resins.

\section{REFERENCES:}

[1] http://www.formula1dictionary.net/ground_effect.html February 2013

[2] http://books.mcgrawhill.com/EST10/site/spotlight/automobiles/articles/Race -CarAerodynamics.pdf February 2013

[3] ASHRAE. 1991. Method of testing for rating the performance of air outlets and inlets,

ANSI/ASHRAE Standard 70 - 1991, Atlanta: ASHRAE.

ASHRAE. 1997. ASHRAE Handbook Fundamentals, ASHRAE, Atlanta, Ch.31.

[4] CHAM. 1998. PHOENICS Version 3.1, CHAM Ltd., London, UK. 
[5] Chen, Q., and Jiang, Z. 1996. Simulation of a complex air diffuser with CFD technique, Proc. Of ROOMVENT '96, Vol. 1, pp. 227-234.

[6] Li, Z.H., Zhang, J.S., Zhivov, A.M., and Christianson, L.L. 1993. Characteristics of diffuser air jets and airflow in the occupied regions of mechanically ventilated rooms - a literature review, ASHRAE Trans., 99(1): 1119-1127.

[7] Knappmiller, K.D. 1994. The mixing and separation characteristics of cold air ceiling jets, Ph.D.Dissertation, Colorado State University, Fort Collins, Colorado.

[8] Heikkinen, J. 1991. Modeling of a supply air terminal for room air flow simulation, Proc. of $12^{\text {th }}$ AIVC Conference, Vol. 3: 213-230. 